\title{
Stress Fracture of the Fibular Sesamoid Bone of the Foot in Cricket: An Unusual Injury in Bowlers
}

\author{
${ }^{1}$ Himmat Dhillon, ${ }^{2}$ Prateek Behera, ${ }^{3}$ Sharad Prabhakar, ${ }^{4}$ Sidak Dhillon, ${ }^{5}$ Mandeep S Dhillon
}

\begin{abstract}
An unusual case of fibular sesamoid stress fracture in the back foot of a bowler is presented. The diagnosis was delayed due to the lack of awareness of the support medical staff of the cricketer. Special radiological views and computed tomographic scans confirmed the diagnosis; nonoperative measures cured the pain, but this recurred on subsequent bowling activities. The current player gave up the game, but the literature supports operative intervention in cases that fail conservative regimens. This case is presented to highlight the occurrence of this rare injury in fast bowlers, and we recommend careful examination of the foot when it is the trailing foot, because this has significant forefoot dorsiflexion during the stance phase.
\end{abstract}

Keywords: Cricket injury, Fast bowler, Fibular sesamoid, Sesamoid fracture, Stress fracture.

How to cite this article: Dhillon $\mathrm{H}$, Behera P, Prabhakar S, Dhillon S, Dhillon MS. Stress Fracture of the Fibular Sesamoid Bone of the Foot in Cricket: An Unusual Injury in Bowlers. J Foot Ankle Surg (Asia-Pacific) 2016;3(1):59-62.

Source of support: Nil

Conflict of interest: None

\section{INTRODUCTION}

Sesamoid bones are found in the substance of tendons or muscles, and these improve the biomechanical function of the tendons that they are associated with. The patella and the pisiform are the two sesamoid bones that are widely known to clinicians. Little mention is made of the existence of sesamoid bones under the great toe (hallux). Two sesamoid bones are present in association with the first metatarsophalangeal (MP) joint of the foot, which contribute significantly to weight transfer and become

\footnotetext{
${ }^{1}$ Physiotherapist, ${ }^{2}$ Senior Resident, ${ }^{3}$ Assistant Professor ${ }^{4}$ Trainee, ${ }^{5}$ Professor

${ }^{1}$ Department of Physiotherapy, Flinders University, Adelaide Australia

2,3,5 Department of Orthopedics, Postgraduate Institute of Medical Education and Research, Chandigarh, India

${ }^{4}$ Department of Orthopedics, Government Multispeciality Hospital, Chandigarh, India
}

Corresponding Author: Prateek Behera, Senior Resident Department of Orthopedics, Postgraduate Institute of Medical Education and Research, Chandigarh, India, Phone: 911722745211, e-mail: pbehera15@gmail.com important in athletes using the ball of the foot during the game, like bowlers who pivot on the foot that they land on. The medial side of the foot plays an essential role in weight bearing, and the first MP joint is directly involved in the weight bearing process, ${ }^{1,2}$ especially when there is a rotational element associated. As the proximal phalanx has a relatively small articular facet, it does not provide enough stability during weight bearing; this is supplemented by the sesamoid complex of the great toe, allowing the MP joint to bear around 40 to $60 \%$ of the body weight during walking., ${ }^{1,2}$

From an anatomical point of view, the hallucial sesamoids are found in the tendons of the flexor hallucis brevis. There are two sesamoid bones which are named as tibial (medial) sesamoid and fibular (lateral) sesamoid. ${ }^{1-3}$ While the tibial sesamoid has attachment from abductor hallucis, the fibular sesamoid has attachment from the adductor hallucis. The two sesamoids are in turn connected by intersesamoid ligament, and they conjointly function as shock absorbers during weight bearing. They tend to decrease friction and thus protect the flexor hallucis brevis tendons. They also increase the moment of the flexor hallucis brevis, which enables plantarflexion of the hallux. Additionally, they elevate the first metatarsal head, which leads to dissipation of the forces on the metatarsal head. ${ }^{3-5}$

In view of the essential role played by the sesamoids in weight bearing, it is not uncommon to find injuries of sesamoids in athletes. The tibial sesamoid is more commonly involved of the two, as it bears greater amount of weight compared with the fibular one. Acute fractures resulting from a direct impact, and stress fractures resulting from repetitive stress have been reported. ${ }^{1,3,4,6}$ Sesamoid stress fractures comprise approximately 5\% of all foot stress fractures, with the medial being more commonly involved. These fractures have perhaps the greatest tendency for nonunion and can result in incapacitating pain on athletic activity. ${ }^{7}$

Any type of injury to the fibular sesamoid is uncommon, and there is very little literature available on the topic. To the best of our knowledge, no case of such an injury associated with cricket has been published. We report the case of a fast bowler who sustained fracture of the fibular sesamoid, presumed to be a stress fracture, 
and was managed conservatively. This ultimately made him leave the game.

\section{CASE REPORT}

An 18-year-old right-handed fast bowler, district-level player, was presented with complaints of pain in the sole of his right foot, in the region of neck of the first metatarsal for the past 1 year. He was asymptomatic at rest; pain was triggered by his run-up for bowling, and maximum intensity of pain was noted immediately after delivering the ball. The right foot was involved, and the pain was also increased when he swiveled for bowling, in the trailing foot, with dorsiflexion of the toe. He specifically denied any significant trauma to the foot, or any blow; the pain had started insidiously, and it had become worse in the 6 weeks prior to his presentation, interfering with his bowling. On clinical examination, no appreciable swelling was noted, but palpation revealed tenderness localized to the lateral aspect of the neck of the first metatarsal. Anteroposterior, lateral, and oblique radiographs of the foot were obtained focusing on the first MP joints; these revealed a healed fracture of the fibular sesamoid, with some irregularity, looking like a bipartite sesamoid (Figs 1A and B). Radiographs of both feet and special sesamoid views were obtained and compared to rule out a bi-partite sesamoid. A computed tomographic (CT) scan was obtained, and this confirmed our diagnosis (Figs $2 \mathrm{~A}$ and $\mathrm{B}$ ). He was given a local infiltration of $2 \%$ lignocaine, which relieved him of his pain, thus confirming that the pain was originating from the malunited fibular sesamoid. In addition, shoe-wear modification by addition of a molded silicone metatarsal pad was suggested, which also helped to maintain the pain relief. The bowler was put into a program of aggressive cross-training. At 4 weeks of follow-up, he was allowed to start jogging. At 4 months follow-up, he became asymptomatic and was asked to resume his cricket practice. Subsequently, however, the pain returned, and he was advised a bone scan with the option of surgery. This he declined, and after 6 months of on and off pain, he gave up the game and settled for college studies.

\section{DISCUSSION}

The increase in intense sporting activity has resulted in greater number of sports-related injuries being reported. In addition, the availability of better imaging modalities has contributed to diagnosis of injuries that were probably not picked up previously.

The two sesamoid bones in the tendon of flexor hallucis brevis are involved in bearing weight and hence can be injured by acute trauma or by repetitive stress. The hallux sesamoids have been reported to be involved in $9 \%$ of foot injuries. ${ }^{4}$ The available literature on hallucial sesamoid injuries in sportsmen is suggestive of track and field athletes being the common sufferers, ${ }^{8-10}$ with the tibial sesamoid more commonly injured. ${ }^{6,11}$ The fibular sesamoid is rarely involved. Christensen et $\mathrm{al}^{12}$ were the first to report a case of fracture of the fibular sesamoid of hallux.

Very limited scientific literature is available on the epidemiological aspects of lower limb injuries in cricketers from India, Pakistan, Bangladesh, or Sri Lanka. The available literature is from South Africa, ${ }^{13,14}$ Australia, ${ }^{15}$ New Zealand, ${ }^{16}$ West Indies. ${ }^{17}$ Cricket is a popular sport in the Indian subcontinent.

Owing to the regular run-up in which the fast bowlers have to accelerate from rest and then deliver a ball, they are vulnerable to foot and ankle injuries. The two sesamoids of the hallux may thus be stressed or injured in bowlers, but there are no available publications documenting this. Ballerinas ${ }^{6}$ and athletes ${ }^{11}$ may get these injuries due to extremes of toe dorsiflexion, but the mechanisms in the foot of bowlers have not been studied. These injuries
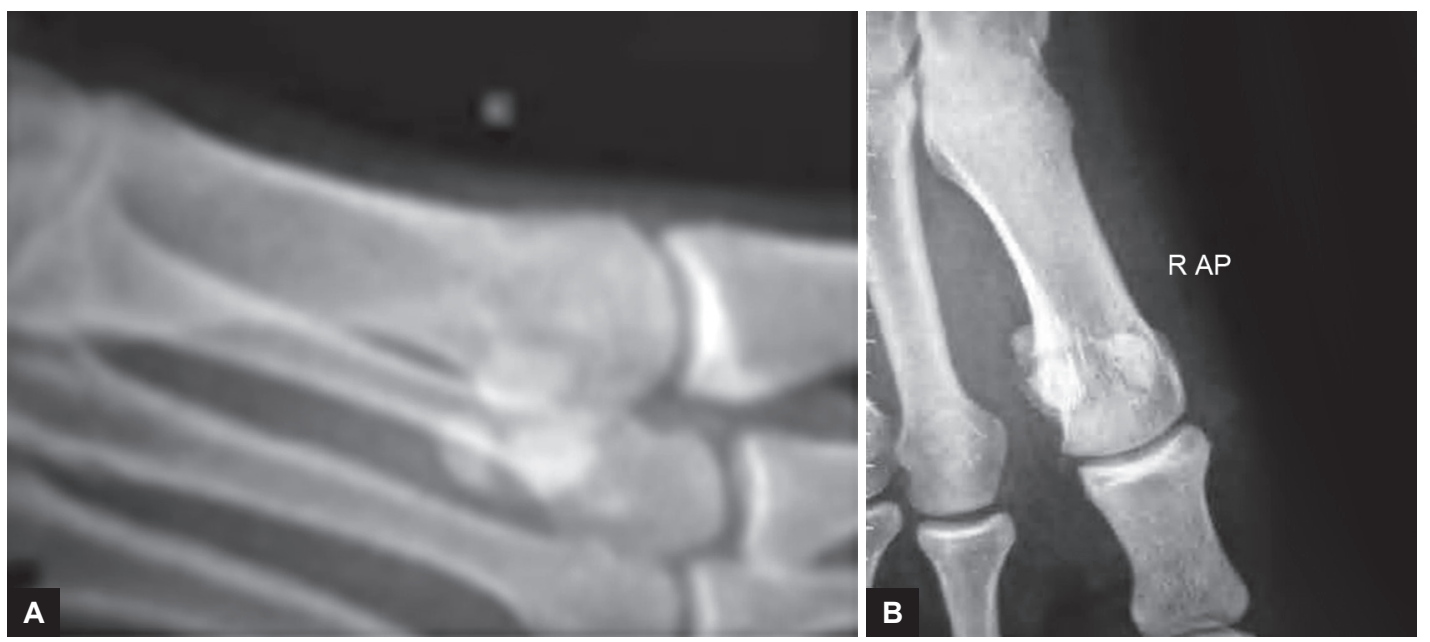

Figs 1A and B: (A) Radiographs showing fracture of fibular sesamoid; and $(B)$ the bone in two parts 

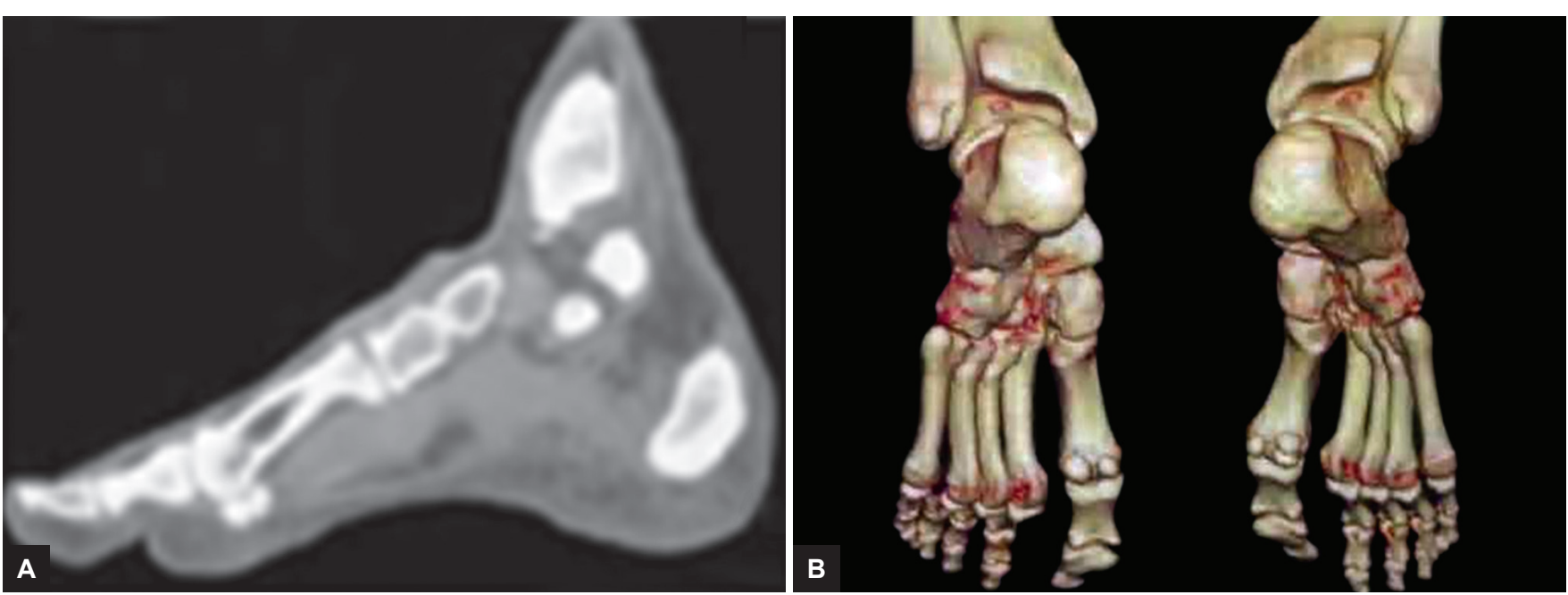

Figs 2A and B: (A) Computed tomographic scan (sagittal cut) of the fracture; and (B) Computed tomographic scan (three-dimensional reconstruction) showing malunion of the fracture

may go unnoticed, and the bowler may continue to play through pain, thus affecting the performance.

Patients presenting with complaints of pain in the region of the neck of the hallux may have suffered an injury to the metatarsal, the proximal phalanx, the MP joint, or the sesamoids. Differentiating first MP pain due to sesamoid pathology from these other disorders can be difficult because the signs and symptoms may be similar. ${ }^{1}$ Signs and symptoms most often include swelling and pain, specifically at the ball of the foot underlying the first MP joint.

The sesamoids may become painful due to acute or chronic trauma, infection, inflammation, and infection. ${ }^{1}$ Traumatic conditions such as fractures or fracture and dislocations can be diagnosed if imaging modalities are used judiciously. Radiographs are readily available, but they may not pick up relatively undisplaced fractures and stress fractures. Symptomatic patients should be evaluated with weight bearing anteroposterior, oblique, and lateral views of the foot and axial sesamoid radiographic projections. Computed tomographic scans, magnetic resonance imaging, and bone scans are much more sensitive in picking up undisplaced or minimally displaced injuries. Radiographs can present a diagnostic dilemma for the clinician as bipartite sesamoids may resemble acute fractures and finding bipartite sesamoids is not uncommon. Bipartite and multipartite sesamoid fragments have smooth, narrow, sclerotic margins, while an acutely fractured sesamoid has a nonsclerotic, irregular margin. ${ }^{1}$ Also, comparative radiographs of the opposite foot are helpful as these are bilateral in most of the cases. Sesamoid fractures are difficult to treat due to the poor blood supply to these bones. The branches of medial plantar artery are the primary source of blood supply.

The optimum choice of treatment for sesamoid fractures is unclear. Treatment is primarily nonsurgical; because pathology is related to increased dorsiflexion of the hallux, treatment aims at preventing this movement while allowing the stress fracture to heal. Initially, conservative treatment by providing rest or partial weight bearing, cessation of sports, application of a short-leg walking cast, or the use of a molded well-padded orthosis for 6 to 8 weeks supported by anti-inflammatory drugs is recommended. ${ }^{18}$

The option for surgical management is offered only in intractable cases when conservative management fails. Surgical options include an open reduction and fixation with screws with or without bone grafts, percutaneous screw fixation, or shaving or sesamoidectomy. ${ }^{1,17}$ Partial sesamoidectomy is often the preferred and practical solution, and the cricketer may return to practice within 3 weeks of this procedure; partial medial sesamoidectomy may also be accomplished arthroscopically. Total sesamoidectomy needs to be avoided as it would produce a mechanical defect in the flexor hallucis brevis tendonmuscle unit and reduce the flexion movement arm of the muscle at the MP joint. ${ }^{19,20}$

In the current case, the bowler had continued to bowl with pain and without any treatment, thus delaying diagnosis. The picture was probably compounded by pain being on the lateral aspect, originating from the fibular sesamoid, which is an uncommon bone to get injured.

The radiology of the case was suggestive of a malunited fracture of the fibular sesamoid, thus implying that even without treatment the fractured sesamoids may actually heal and unite. But the malunion becomes painful after continuing insult, and this was the case with this fast bowler. The diagnosis was confirmed when the local infiltration of Lignocaine brought pain relief to the patient. We successfully relieved the pain by foot-wear modification, and after gradual training, the bowler was able to return to cricket. Unfortunately, 
the pain recurred, and when offered surgery, the player chose to give up cricket and concentrate on his college studies and a future job.

The extent of dorsiflexion of the toes on the planted foot of fast bowlers during the delivery stride needs to be evaluated, especially when they swivel on it. This has a lot of potential for future research, as impacts on landing and excessive dorsiflexion of the toe may be causative factors of acute or stress-related injuries of the sesamoids.

\section{CONCLUSION}

Fibular sesamoid stress fracture is uncommon and may not come to mind immediately in players with medial foot pain. Fast bowlers tend to over strain the great toe dorsiflexion, and unremitting pain related to activity should arouse suspicion in treating clinicians. Specific radiology clinches the diagnosis; most treatment focuses on nonoperative measures, and if initiated early, it seems to be successful. Patients with recurrence of pain need bone scans and probable surgery.

\section{REFERENCES}

1. Patel T, Song AJ, Lomasney LM, Demos TC, Dickey S. Acute fibular sesamoid fracture: one part of the spectrum of sesamoid pathologies. Orthopedics 2014 Oct;37(10):650-711.

2. Awh MH. MRI web clinic: turf toe [cited 2015 Aug]. http:// www.radsource.us/clinic/0303.

3. Cohen BE. Hallux sesamoid disorders. Foot Ankle Clin 2009 Mar;14(1):91-104.

4. Demond BT, Cory JW, McBryde A. The hallucal sesamoid complex. J Am Acad Orthop Surg 2006;14(13):745-753.

5. Leventen EO. Sesamoid disorders and treatment. Clin Orthop 1991;269:236-240.
6. Biedert R, Hinterman B. Stress fractures of the medial great toe sesamoid in athletes. Foot Ankle Int 2003;24(2):137-141.

7. Ezequiel, P.; Eran, K.; Eugene, K.; Niv, M.; Iftach, H.; Meir, N.; Gideon, M. Stress fractures of the foot in footballers. Volpi, P. editor. Football traumatology. Switzerland: Springer International Publishing; 2015. p. 371-383.

8. Richardson EG. Injuries to the hallucal sesamoids in the athlete. Foot Ankle 1987 Feb;7(4):229-244.

9. Iwamoto J, Takeda T. Stress fractures in athletes: review of 196 cases. J Orthop Sci 2003;8(3):273-278.

10. Murray SR, Reeder MT, Udermann BE, Pettitt RW. High-risk stress fractures: pathogenesis, evaluation, and treatment. Compr Ther 2006 Spring;32(1):20-25.

11. Burton EM, Amaker B. Stress fracture of the great toe sesamoid in a ballerina: MRI appearance. Pediatr Radiol 1994;24:37-38.

12. Christensen SE, Cetti R, Niebuhr-Jørgensen U. Fracture of the fibular sesamoid of the hallux. Br J Sports Med 1983 Sep;17(3): 177-179.

13. Stretch RA. The incidence and nature of injuries in club and provincial cricketers. S Afr Med J 1993;83:339-341.

14. Stretch RA. Cricket injuries: a longitudinal study of the nature of injuries to South African cricketers. Br J Sports Med 2003;37:250-253.

15. Orchard JW, James T, Portus MR. Injuries to elite male cricketers in Australia over a 10-year period. J Sci Med Sport 2006 Dec;9(6):459-467.

16. Orchard J, Finch C. Australia needs to follow New Zealand's lead on sports injuries. Med J Aust 2002 Jul1;177(1):38-39.

17. Mansingh A, Harper L, Headley S, King-Mowatt J, Mansingh G. Injuries in West Indies Cricket 2003-2004. Br J Sports Med 2006 Feb;40(2):119-123.

18. Mittlmeier T, Haar P. Sesamoid and toe fractures. Injury. 2004 Sep;35(Suppl 2):SB87-SB97.

19. Aper RL, Saltzman CL, Brown TD. The effect of hallux sesamoid excision on the flexor hallucis longus moment arm. Clin Orthop 1996 Apr;(325):209-217.

20. Richardson EG. Hallucal sesamoid pain: causes and surgical treatment. J Am Acad Orthop Surg 1999 Jul-Aug;7(4): 270-278. 